\title{
The mechanism and kinetics of void formation and growth in particulate filled PE composites
}

\author{
A. Sudár1,2, J. Móczón ${ }^{1,2}$, Gy. Vörös ${ }^{1,3}$, B. Pukánszky1,2* \\ ${ }^{1}$ Laboratory of Plastics and Rubber Technology, Department of Physical Chemistry and Material Science, \\ Budapest University of Technology and Economics, H-1521 Budapest, P.O. Box 91, Hungary \\ ${ }^{2}$ Institute of Materials and Environmental Chemistry, Chemical Research Center, Hungarian Academy of Sciences, \\ H-1525 Budapest, P.O. Box 17, Hungary \\ ${ }^{3}$ Department of Materials Physics, Eötvös University Budapest, H-1518 Budapest, P.O. Box 32, Hungary
}

Received 3 July 2007; accepted in revised form 4 October 2007

\begin{abstract}
Volume strain measurements were carried out on $\mathrm{PE} / \mathrm{CaCO}_{3}$ composites prepared with three different matrix polymers, containing various amounts of filler. The analysis of the debonding process and the various stages of void formation proved that the model developed for the prediction of the initiation of debonding is valid also for the studied $\mathrm{PE} / \mathrm{CaCO}$ composites. Debonding stress is determined by the strength of interfacial adhesion, particle size and the stiffness of the matrix. In thermoplastic matrices usually two competitive processes take place: debonding and the plastic deformation of the polymer. The relative magnitude of the two processes strongly influences the number and size of the voids formed. Because of this competition and due to the wide particle size distribution of commercial fillers, only a certain fraction of the particles initiate the formation of voids. The number of voids formed is inversely proportional to the stiffness of the matrix polymer. In stiff matrices almost the entire amount of filler separates from the matrix under the effect of external load, while less than $30 \%$ debond in a PE which has an initial modulus of $0.4 \mathrm{GPa}$. Further decrease of matrix stiffness may lead to the complete absence of debonding and the composite would deform exclusively by shear yielding. Voids initiated by debonding grow during the further deformation of the composite. The size of the voids also depends on the modulus of the matrix. The rate of volume increase considerably exceeds the value predicted for cross-linked rubbers. At the same deformation and filler content the number of voids is smaller and their size is larger in soft matrices than in polymers with larger inherent modulus.
\end{abstract}

Keywords: polymer composites, particulate filled polymers, debonding, growth of voids, shear yielding

\section{Introduction}

Particulate filled polymers are used in large quantities in many fields of application. They are extensively applied in the automotive industry as bumpers, various panels and structural components, as well as under hood parts [1]. Garden furniture and household articles are also prepared from them and they are applied in construction as well $[1,2]$. One particular application is the production of breathable films, which allow the passage of vapor, but prevent the permeation of liquids. These films are utilized as the back sheets of diapers, in other sanitary applications and as internal roof insulation [2, 4]. In heterogeneous polymer systems, stress concentration develops around the particles under the effect of external load and the actual stress distribution determines the local micromechanical deformation processes [5]. In particulate filled polymers the dominant deformation mechanism is the separation of the matrix/filler interface, i. e. debonding, which leads to a volume increase during deformation [6]. The preparation of 
breathable films is based on and utilizes this process. Films are produced from polyethylene containing a large amount, $40-50 \mathrm{wt} \% \mathrm{CaCO}_{3}$ and they are stretched to create the porous structure, which makes breathing possible. However, the size of the voids developing during debonding must be controlled otherwise the film does not fulfill its role. Large holes let also the liquid pass through the film, while the moisture vapor transmission rate (MVTR) of the films is not sufficiently large if very small voids are created. An optimization of component characteristics and process parameters is necessary to achieve films offering maximum performance, i. e. as large MVTR (moisture vapor transmission rate) as possible accompanied by acceptable mechanical properties. Optimization requires the detailed analysis of the debonding process and the factors determining it.

The properties, including vapor transmission, of breathable films are determined by the number and size of the voids formed during their deformation, which, in their turn, depend on the initiation and growth of the voids. The debonding process, which takes place during the deformation of particulate filled polymers, was studied earlier by several authors [7-16]. Models were developed, which predict the conditions for the initiation of debonding $[6,17,18]$, attempts were made to predict the number of debonded particles $[8,19]$ and the growth of voids was also described in a paper [7]. However, a concise analysis of the debonding process has never been carried out in the past. The goal of our work was to analyze the separation of matrix/filler interface in polyethylene composites containing various amounts of $\mathrm{CaCO}_{3}$ filler. Debonding was determined by the measurement of volume increase during deformation and the results were critically analyzed in view of existing theories. The most important factors governing void formation, as well as the number and size of the created particles are pointed out as a result of the analysis.

\section{Background}

The most important characteristic of breathable films is MVTR, which depends on the number and size of voids forming during the stretching process. The initiation of debonding is predicted by models $[6,17,18]$, but for ideal conditions. In practice, only a part of the particles initiate void formation, their number must be estimated from the results of measurements with the help of appropriate theories. Finally, the created voids increase during further deformation, their size depends on the extent of stretching and on some other factors [7]. In this section a brief account is given about approaches which were developed to predict the three main aspects of the debonding process.

\subsection{Initiation}

According to our knowledge, two models exist for the prediction of the stress necessary to initiate debonding, those developed by Vollenberg $[17,18]$ and Vörös [20], respectively. According to both models initiation stress depends on the strength of interfacial interaction, on the size of the particles and on the stiffness of the matrix (Equation (1)) [20]:

$$
\sigma_{D}=-C_{1} \sigma_{T}+C_{2} \sqrt{\frac{W_{A B} E}{R}}
$$

where $\sigma_{D}$ and $\sigma_{T}$ are debonding and thermal stress, respectively, $W_{A B}$ the reversible work of adhesion, $E$ the Young's modulus of the matrix, $R$ the radius of the particles, while $C_{1}$ and $C_{2}$ are constants. Accordingly, larger initiation stress is needed for debonding to start with increasing adhesion of the components, with increasing stiffness of the matrix and with decreasing particle size. The effect of particle size and interfacial adhesion on debonding stress was studied extensively in the past and results unambiguously proved the validity of the model $[6,20]$. As a consequence, our attention is focused on the influence of matrix modulus on the debonding process in this paper.

\subsection{The number of debonded particles}

The model leading to Equation (1) was developed by using certain conditions, most of which are not fulfilled in practice. Debonding stress was derived for a single particle embedded in an infinite matrix $[17,18,20]$. However, the particle size distribution of commercial fillers is relatively wide; we cannot expect voids to form around all the particles. According to Equation (1) large particles debond easily, while small ones remain strongly attached to the matrix. The picture is further complicated by the fact that small particles form aggregates [2125], which behave as large particles and debond 
under the effect of external stress forming very large holes [26]. The number of debonded particles can be estimated by model calculations. The approach assumes that a part of the filler particles is strongly bonded to the matrix, carries load and increases stiffness, while the rest debonds and does not contribute to the increase of modulus. Accordingly, the composition dependence of Young's modulus, $E(\varphi)$, can be expressed by the KernerNielsen equation (2) [27, 28]:

$E_{c}(\varphi)=E_{m} \frac{1+A_{f} B_{f} \varphi_{b}}{1-\Psi_{f} B_{f} \varphi_{b}} \frac{1-\Psi_{d} B_{d} \varphi_{d}}{1+A_{d} B_{d} \varphi_{d}}$

where $E_{m}$ is the modulus of the matrix polymer, $\varphi_{b}$ and $\varphi_{d}$ are the volume fraction of bonded and debonded particles, respectively. In this form the model assumes that voids form around particles in a matrix containing the filler in $\varphi_{d}$ volume fraction. $A_{f}$ and $A_{d}$ take the Equations (3) and (4):

$A_{f}=\frac{7-5 v_{m}}{8-10 v_{m}}$ and $A_{d}=\frac{8-10 v_{m f}}{7-5 v_{m f}}$

where $v_{m}$ and $v_{m f}$ are the Poisson's ratio of the neat matrix and that containing the bonded particles, respectively. Parameter $B_{f}$ and $B_{d}$ can be calculated from Equations (5) and (6):

$B_{f}=\frac{\frac{E_{f}}{E_{m}}-1}{\frac{E_{f}}{E_{m}}+A_{f}}$ and $B_{d}=\frac{\frac{E_{m f}}{E_{d}}-1}{\frac{E_{m f}}{E_{d}}+A_{d}}$

Since the modulus of the voids is zero, $B_{d}$ assumes the value of $1 . \Psi$ is a correction factor taking into account the maximum packing fraction $\left(\varphi_{\max }\right)$ of the inclusion and it takes the same form for bonded and debonded particles (Equation (7)):

$\Psi_{f}=1+\left(\frac{1-\varphi_{\text {bmax }}}{\varphi_{b \max }^{2}}\right) \varphi_{b}$

Naturally always the appropriate volume fraction and $\varphi_{\max }$ value must be introduced into the equation. Finally, the following correlation can be writ- ten for the relation of the various filler volume fractions (Equation (8)):

$\varphi_{b}=\varphi_{f}-\varphi_{d}$

where $\varphi_{f}$ is total filler content, while $\varphi_{d}$ is the volume fraction of debonded particles. If we determine the modulus $\left(E_{c}\right)$ of prestrained composite samples and know $v_{m}$, the amount of bonded $\left(\varphi_{b}\right)$ and debonded $\left(\varphi_{d}\right)$ particles can be calculated from Equation (2).

\subsection{Size of the voids}

Voids initiated by the separation of the matrix/filler interface grow during further elongation. Farris [7] carried out his analysis for a single particle and assumed that each void formed around it is an ellipsoid of revolution with the two equal minor axes fixed by the diameter of the particle. During deformation the major axis of the void increases linearly with strain at a rate proportional to the size of the contained particle. Accordingly, the rate of volume increase, thus the size of the formed voids, depends on filler content in the following way (Equation (9)):

$$
\frac{\mathrm{d}(\Delta V)}{\mathrm{d} \varepsilon}=C \varphi
$$

where $\Delta V$ is volume increase, $\varepsilon$ deformation, $\varphi$ the volume fraction of the filler and $C$ is a constant. Farris [7] carried out his experiments in crosslinked polyurethane rubbers and claimed that parameter $C$ is close to 1 for particles with a uniform size. In subsequent sections we analyze our experimental results by using the models presented above and identify the most important factors which determine the debonding process and finally the MVTR of breathable films.

\section{Experimental}

Three polyethylene grades with different stiffnesses were used in the experiments. The most important characteristics of the polymers are listed in Table 1. The $\mathrm{CaCO}_{3}$ filler added to the polymers

Table 1. Characteristics of the PE matrix polymers used in the study

\begin{tabular}{|c|l|c|c|c|c|}
\hline Abbrev. & \multicolumn{1}{|c|}{ Trade name } & Producer & MFI [g/10 min] & Density [g/cm $\left.{ }^{3}\right]$ & Modulus [GPa] \\
\hline PE04 & Dowlex 2037 & Dow & 2.5 & 0.935 & 0.4 \\
\hline PE07 & Tipolen FB 472 & TVK & 0.7 & 0.947 \\
\hline PE11 & Tipolen ME 610 & TVK & 6.5 & 0.961 \\
\hline
\end{tabular}


was the Omyacarb $2 \mathrm{GU}$ grade, a product of Omya $\mathrm{GmbH}$, Switzerland. The average particle size of the filler is $2.5 \mu \mathrm{m}$ and its specific surface area is $3.6 \mathrm{~m}^{2} / \mathrm{g}$. The filler was used without any coating. The filler content of the composites changed from 0 to 0.3 volume fraction in 0.05 volume fraction steps.

The composites were homogenized in a Brabender DSK 42/7 twin-screw compounder at 180-190$200^{\circ} \mathrm{C}$ and $50 \mathrm{rpm}$. Injection molded tensile bars of $150 \times 10 \times 4 \mathrm{~mm}$ dimensions were produced from the pellets using a Battenfeld BA $200 \mathrm{CD}$ machine. The composites were processed at 160-180$200^{\circ} \mathrm{C}$, the temperature of the mold was kept at $40^{\circ} \mathrm{C}$, while injection pressure was 50 bar, holding pressure 45 bar and holding time $15 \mathrm{sec}$.

The tensile properties of the composites were derived from force vs. elongation traces recorded by using an Instron 5566 machine with a cross head speed of $50 \mathrm{~mm} / \mathrm{min}$. Volume strain was determined by measuring also the change in one lateral dimension of the specimen with a strain transducer. Volume strain measurements were carried out at $5 \mathrm{~mm} / \mathrm{min}$ cross-head speed. In order to avoid complications in the evaluation of the results possibly caused by the inhomogeneous deformation of the specimens, volume strain measurements were always interrupted at the onset of macroscopic yielding $[29,30]$. We assumed that changes in both lateral directions are the same. In order to determine the number of debonded particles, specimens were prestrained to different extents. Strained samples were allowed to relax for $15 \mathrm{~min}$ and their Young's modulus was determined at $0.5 \mathrm{~mm} / \mathrm{min}$ cross-head speed, i. e. 1\% deformation rate. All measurements were done at room temperature.

\section{Results and discussion}

The various stages of void formation, i. e. debonding and growth are discussed separately in this section. First the main factors determining the initiation of the voids are presented briefly, and then the number of debonded particles is estimated as described above. The rate of void growth and the final void content of the composites are discussed in the last subsection.

\subsection{Initiation}

The main factors determining the initiation of debonding is specified by Equation (1). The validity of the correlation was proved several times both by Vollenberg [17] and Pukánszky et al. [6], who showed that interfacial adhesion and particle size influence debonding stress in the way described by the model [6]. Less attention was paid to the effect of matrix stiffness. Stress vs. strain, as well as volume strain traces of composites with $20 \mathrm{vol} \%$ filler content are presented in Figure 1 for the three polyethylene grades investigated in this study. The stiffness of the matrix polymer strongly influences the modulus, yield stress and yield strain of the composites, the first two decreases, while the last increases with decreasing stiffness, as expected. The differences in volume strain are less unambiguous. The largest volume increase is shown by the softest matrix, while the volume strain of the other two composites is very similar to each other. Initiation stress $\left(\sigma_{D}\right)$ was determined as the intersection of the first, almost horizontal part and the increasing section of the volume strain traces, and it is plotted against the stiffness of the matrix in Figure 2 in the form indicated by Equation (1). Although only three polymers were included into the study, the correlation presented in Figure 2 strongly supports the model; debonding stress increases linearly with the square root of matrix modulus. Figure 2 supplies an additional proof for the validity of the debonding model developed earlier, thus it can be used for the prediction of the number of voids. Unfortunately, commercial fillers

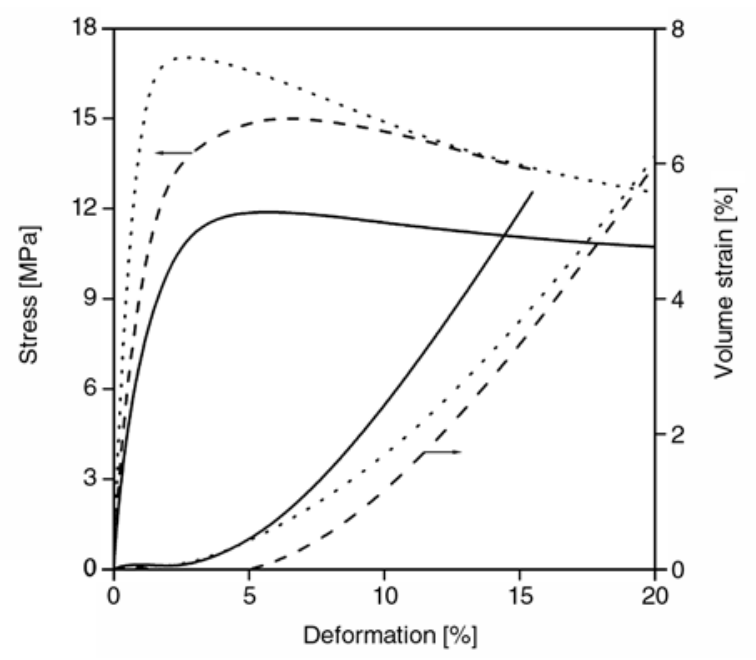

Figure 1. Stress vs. strain and volume strain traces of $\mathrm{PE}$ composites containing $20 \mathrm{vol} \% \mathrm{CaCO}_{3}$ filler. - PE04, - - PE07, . . . . PE11 


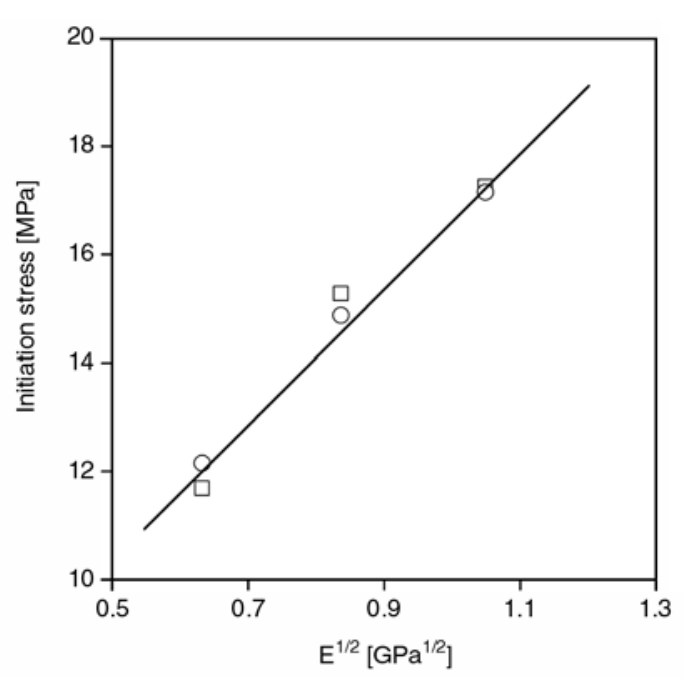

Figure 2. Dependence of debonding stress derived from the volume strain measurements on the stiffness of the matrix (see Equation (1)). Symbols:

(ㅁ) $15 \mathrm{vol} \%$, (o) $20 \mathrm{vol} \%$ filler

have a wide particle size distribution, which may complicate prediction. Nevertheless, we may assume that besides initiation stress the modulus of the matrix polymer probably influences also the number of debonded particles.

\subsection{Extent of debonding}

Volume strain traces are presented for the softest matrix containing various amounts of filler in Figure 3. It is obvious from the figure that volume increases both with filler content and with the extent of elongation. At the largest filler loading the samples broke already after limited extension.

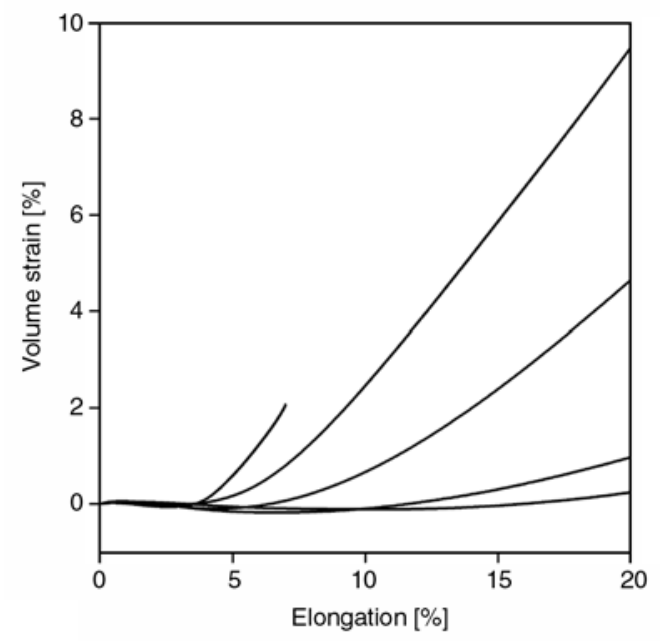

Figure 3. Effect of filler content on volume increase in $\mathrm{PE} 04 / \mathrm{CaCO}_{3}$ composites. Filler content increases from 0.1 to 0.3 volume fraction in 0.05 volume fraction steps from right to left
Although volume increases with filler content, the extent of debonding cannot be estimated from Figure 3. The comparison of the various traces indicates a nonlinear relationship between volume increase and filler content, which may be caused both by the changing number of debonded particles and by the varying rate of volume increase. Very small volume increase occurs at low filler content; in fact volume strain could not be measured reliably at $5 \mathrm{vol} \%$. We can conclude, though, that the recorded volume strain traces do not make possible the estimation of the number of debonded particles, this can be determined only by additional experiments.

The modulus of prestrained composite samples is presented in Figure 4. All three matrices contained the filler in $20 \mathrm{vol} \%$. The shape of the correlation is very similar in all three cases, a horizontal or slightly increasing section is followed by a relatively steep decrease. Modulus seems to reach constant, equilibrium value at large prestrains. The modulus of the three composites differs considerably, and the decrease in stiffness as an effect of prestrain seems to be proportional to the initial modulus of the polymer. The correlations presented in Figure 4 obviously support the validity of the Kerner-Nielsen equation [27, 28] assuming that only bonded particles carry load. Nevertheless, the approach merits somewhat more considerations.

In the investigated composites containing PE and uncoated filler, interfacial interaction is created by secondary, van der Waals forces. These forces can be characterized by the reversible work of adhesion

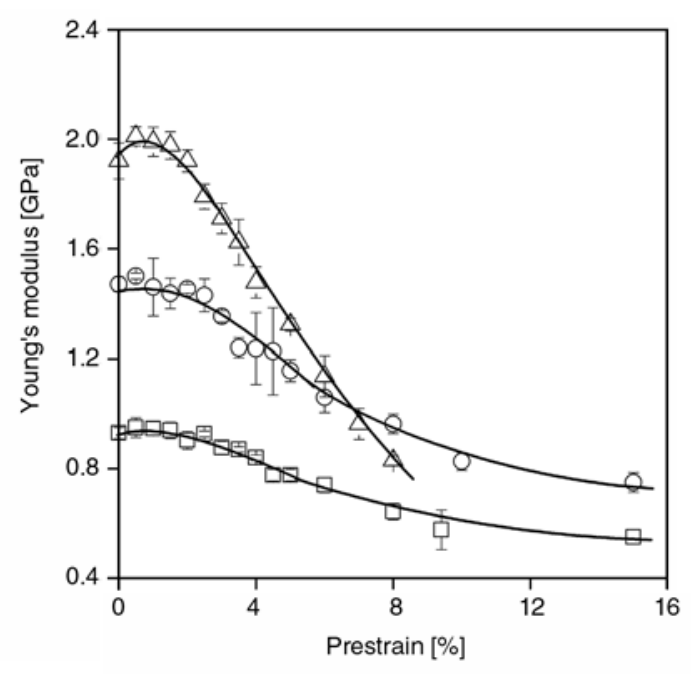

Figure 4. Dependence of the modulus of PE composites containing $20 \mathrm{vol} \%$ filler on the extent of prestrain. Symbols: (口) PE04, (o) PE07, ( $\Delta$ ) PE11 
indicating the reversibility of the debonding process. Although the traces presented in Figure 4 clearly indicate the initiation of debonding, which can be defined as the intersection of the upper horizontal and the declining sections of the correlations presented in Figure 4, debonding may occur already at smaller deformations. Before the determination of modulus specimens were allowed to relax for 15 min, during which the interfacial bond could have reformed completely. Several authors, including Vollenberg et al. [17] and Dubnikova et al. [31] claim that initiation occurs in the increasing part of the stress vs. strain curve (see Figure 1), while Pukánszky et al. [6] found that initiation occurs at the maximum of the curve, i. e. at yielding. Although this question cannot be decided here, we may assume that modulus starts to decrease when the specimen was subjected to sufficient prestrain resulting in limited plastic deformation, which prevents the reformation of the original interfacial bonds. As a consequence, in subsequent sections, we identify the initiation of debonding as the strain at which sufficient plastic deformation occurs, which prevents the reformation of these bonds. These results also imply that debonded particles do not carry load, indeed, and the approach Kerner and Nielsen $[27,28]$ can be used for their estimation. The amount of debonded filler was calculated from the results of Figure 4 by the approach mentioned above (see Equations (2)-(7). The results are presented in Figure 5 for the same composites. The

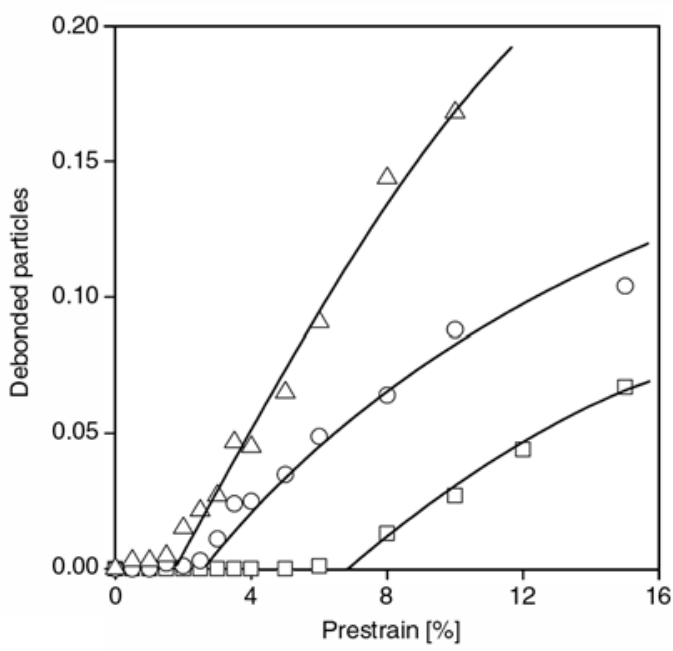

Figure 5. Effect of prestrain and matrix stiffness on the amount (in absolute volume fraction) of debonded filler in $\mathrm{PE} / \mathrm{CaCO}_{3}$ composites containing 20 vol\% filler. () PE04, (o) PE07, $(\Delta)$ PE11 correlation clearly shows the existence of an initiation prestrain, which differs for the three polymers; debonding starts earlier in a stiffer matrix than in a soft polymer. Not only initiation strain or stress, but also the maximum amount of debonded filler differs for the three matrices. The correlations presented in Figure 5 clearly show that in softer matrices only a part of the particles initiate voids, the rest remain bonded to the polymer even at considerably larger deformations than the yield strain of the composite. The character of the correlation and the equilibrium value of the amount of debonded particles indicate that two competitive processes take place during the deformation of these composites: debonding and plastic deformation. The relative magnitude of the two determines the number of voids formed and probably also their size. The calculation of relative amounts indicates that more than $80 \%$ of the particles debond in the stiffest matrix, while this number is about 50 and $30 \%$ for the other two matrices. This shows that the efficiency of void formation is very poor in the softest matrix, less then the half of the particles contribute to the creation of holes. Moreover, since thin films are deformed under plain stress conditions, which results in large plastic deformation, the efficiency of void formation may be even smaller under the conditions of breathable film production. The effect of filler content on the amount of debonded particles is presented in Figure 6 for the softest matrix. At small filler loadings, the determi-

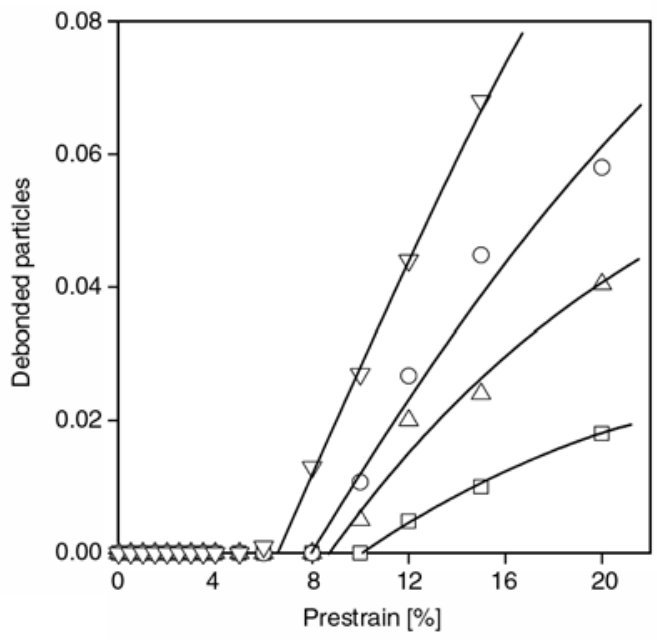

Figure 6. Influence of filler content on the number of debonded particles (in absolute volume fraction) in $\mathrm{PE} 04 / \mathrm{CaCO}_{3}$ composites. Symbols: () $5 \mathrm{vol} \%,(\Delta) 10 \mathrm{vol} \%$, (o) $15 \mathrm{vol} \%$, ( $\nabla) 20$ vol\% filler 
nation of this quantity is very difficult, because of the low modulus of the composites and the small changes in properties during prestraining. In spite of these difficulties, the results allow some interesting observations. Debonding is initiated at almost the same deformation, irrespectively of filler content. Although the absolute amount of debonded particles increases with filler content, the relative amount decreases. This result requires further investigation and analysis. Aggregates may form at larger filler contents, the decrease of effective load bearing cross-section may increase local plastic deformation and the interacting stress fields of neighboring particles might also influence the extent of debonding. We may conclude that matrix stiffness plays a crucial role in the debonding process of particulate filled polymers and the competition between debonding and shear yielding of the matrix determines the actual number of debonded particles. As a consequence, the selection of matrix polymer has a crucial impact on the properties of breathable films, including MVTR and stiffness.

\subsection{Size of the voids}

The final size of the voids depends on the extent of stretching. According to Farris [7] the rate of volume increase is proportional to the filler content of the composites. He found in his experiments that the proportionality constant, $C$, equals 1 . However, he investigated cross-linked rubbers, which do not yield and meet quite well the assumptions of his treatment. Conditions are completely different in thermoplastics, where plastic deformation is considerable. These considerations are strongly supported by Figure 7 showing a SEM micrograph taken from the fracture surface of a composite

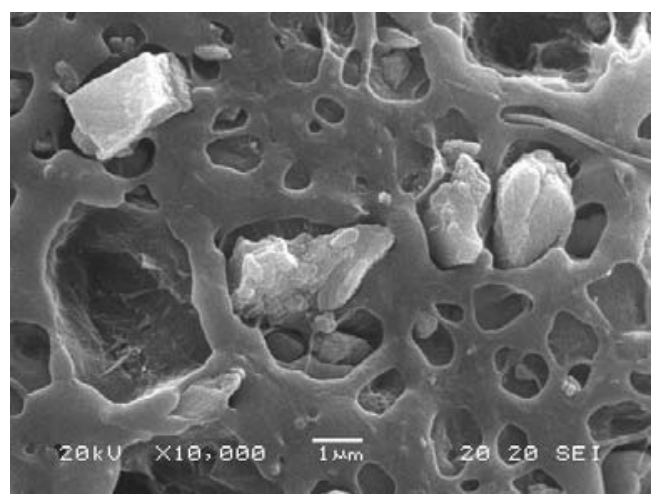

Figure 7. Size and shape of voids forming during elongation in $\mathrm{PE} / \mathrm{CaCO}_{3}$ composites; $5 \varepsilon_{y}$ strain

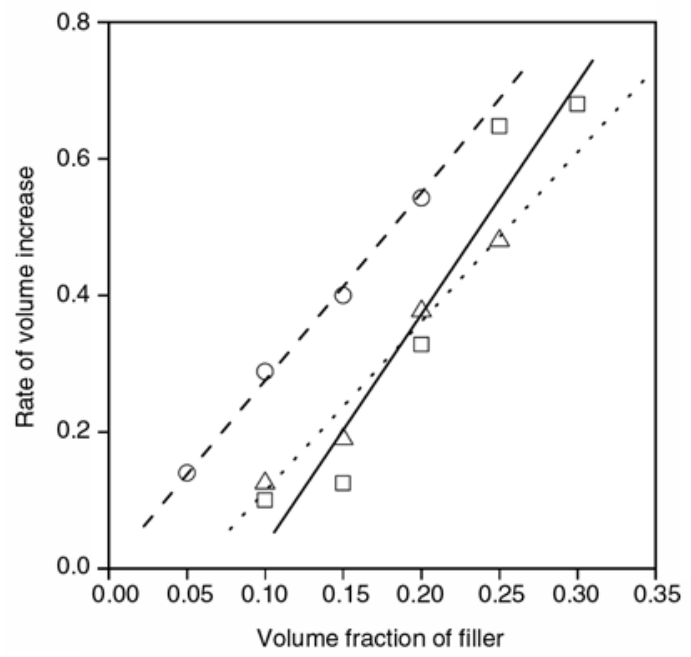

Figure 8. Effect of filler content and matrix stiffness on the rate of volume increase in $\mathrm{PE} / \mathrm{CaCO}_{3}$ composites. Symbols: (口) PE04, (o) PE07, ( $\Delta$ ) PE11

extended to $5 \varepsilon_{y}$ (yield strain). Most of the voids are not regular ellipsoids of revolution and the smaller diameter of the void does not correspond to that of the particle.

The rate of volume increase, i. e. $\mathrm{d}(\Delta V) / \mathrm{d} \varepsilon$ is plotted against filler content in Figure 8. It was determined by fitting a straight line to the increasing part of the volume strain traces (see Figsures 1 and 3). We must call the attention here to the fact that volume strain traces differed considerably from one specimen to the other and the determination of the rate of volume increase was not always easy. As a consequence, the values plotted in the figure must be treated with care. We obtain three different correlations for the three matrix polymers. We can fit straight lines to all three functions, thus they seem to correspond to the prediction of Farris. However, the slopes of the lines differ from each other and also from the predicted value of 1 . The steepest slope was obtained for the softest matrix, while the smallest for PE11. Moreover, the extrapolation of two of the lines would indicate zero volume strain below a certain filler content, which does not agree with the prediction of Farris [7]. Considering the difficulties of determination, the relatively small number of experimental points as well as their considerable scatter, we refrain from further interpretation of the data in order to avoid any unjustified speculation. Further, more detailed experiments must be carried out in order to precisely determine the exact nature of the correlations. However, the tendencies observed are clear and the present results suffice for the purpose of this paper. The 


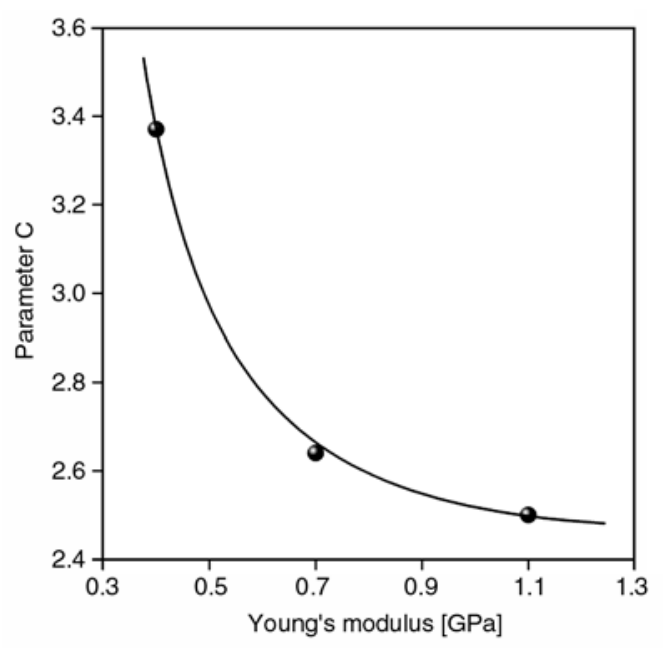

Figure 9. Dependence of parameter $C$ on the stiffness of the matrix polymer

correlation between parameter $C$ and the modulus of the matrix polymer is presented in Figure 9. Obviously, deformation proceeds in a different way in polyethylene than in cross-linked rubber. Although three values are not sufficient for drawing unambiguous conclusions, based on Figure 9 we may assume that even further increase in stiffness would not decrease the value of parameter $C$ to 1 . The large $C$ value indicates that much larger voids form in a thermoplastic matrix than in a rubber. This proves again that competitive processes take place during the deformation in the former case and the size of the voids is influenced also by the extent of plastic deformation. Our statement is strongly corroborated by Figure 9, which shows that a $C$ value of 3.4 was obtained for the softest matrix, while only 2.5 for the stiffest. Considering the results presented in the previous subsection we may conclude that less particles initiate voids in a soft matrix, but their size becomes larger during further growth than in a stiffer polymer.

The influence of plastic deformation on the final void content of the polymer and consequently on permeability is demonstrated well by Figure 10, in which volume increase is presented as a function of filler content at various extents of deformation for PE04. At small strains volume does not increase practically at all and at least 0.2 volume fraction of filler is needed to achieve observable volume increase. In accordance with results presented above, only a small fraction of the filler particles debond in the soft matrix and plastic deformation dominates at small filler content. The correlation explains also the uncertainty encountered during

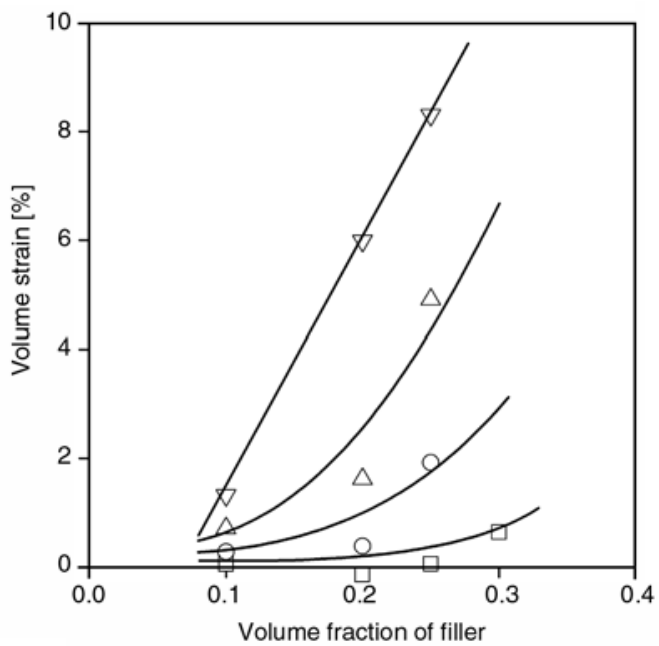

Figure 10. Effect of filler content and strain on volume increase; extensive plastic deformation during the elongation of PE04 composites. Symbols: (口) $5 \%,($ o) $10 \%,(\Delta) 15 \%,(\nabla) 20 \%$ strain

the estimation of the number of debonded filler particles at small filler loadings. With increasing filler content the stiffness of the composite as well as the number of voids increase, which results in considerable volume increase. Volume strain is plotted against filler content for the PE07 composites in Figure 11. All correlations are linear showing a proportional increase of void content with increasing filler content, which indicates that plastic deformation is less significant in this polymer than in the softest one. These results prove that all stages of void formation are equally important in the determination of the moisture permeation of breathable films and only the proper optimization of component properties (matrix stiffness, particle size of the

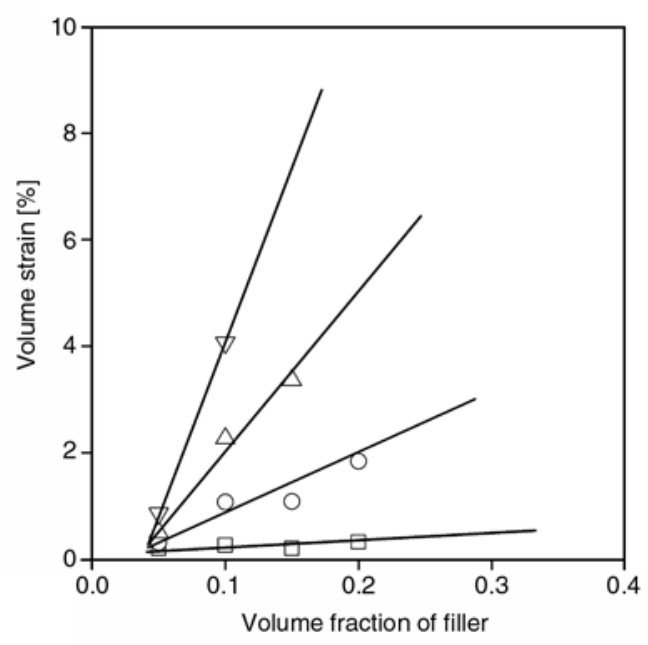

Figure 11. Volume increase in PE07 composites at different filler contents and deformation. Symbols: () $5 \%,($ o) $10 \%,(\Delta) 15 \%,(\nabla) 20 \%$ strain 
filler, interfacial adhesion), composition (filler content) and processing technology (extent and conditions of stretching) may lead to films with acceptable properties.

\section{Conclusions}

The analysis of the debonding process and the various stages of void formation in particulate filled composites proved that the model developed for the prediction of the initiation of debonding is valid also for the studied $\mathrm{PE} / \mathrm{CaCO}_{3}$ composites. Accordingly, debonding stress is determined by the strength of interfacial adhesion, particle size and the stiffness of the matrix. However, in thermoplastic matrices usually two competitive processes take place: debonding and the plastic deformation of the polymer. The relative magnitude of the two processes strongly influences the number and size of the voids formed. Because of this competition and due to the wide particle size distribution of commercial fillers, only a fraction of the particles initiate the formation of voids. The number of voids formed is inversely proportional to the stiffness of the matrix polymer. In stiff matrices almost the entire amount of filler separates from the matrix under the effect of external load, while less then $30 \%$ debond in a PE which has an initial modulus of $0.4 \mathrm{GPa}$. Further decrease of matrix stiffness may lead to the complete absence of debonding and the composite would deform exclusively by shear yielding. Voids initiated by debonding grow during the further deformation of the composite. The size of the voids also depends on the modulus of the matrix. The rate of volume increase considerably exceeds the value predicted for cross-linked rubbers. The results clearly prove that in soft matrices the number of voids is smaller and their size is larger at the same deformation and filler content than in polymers with larger inherent modulus. In order to achieve sufficiently large vapor transmission in breathable films, all parameters of the process must be optimized.

\section{Acknowledgements}

This paper was supported by the Bolyai János Research Scholarship of the Hungarian Academy of Sciences. The authors are indebted to Alpár Farkas and Dénes Horváth MSc students as well as to Richard Twohig guest student for their help in sample preparation and volume strain measurements. The research on heterogeneous polymer systems was partly financed by the National Scientific Research Fund (OTKA Grant No. F 68579); its help is highly appreciated. The authors are grateful for TVK, as well as for Gábor Nagy and his colleagues for providing the polymers for this study.

\section{References}

[1] Rothon R. N.: Particulate-filled polymer composites. Rapra, Shawbury (2003).

[2] Markarian J.: Mineral modifiers take on new roles. Plastics Additives and Compounding, 6, 26-31 (2004).

[3] Morieras G.: Baby boom market for fillers, GCC in microporous films. Industrial Minerals, 6, 29-33 (2001).

[4] Hale W. R., Dohrer K. K., Tant M. R., Sand I. D.: A diffusion model for water vapor transmission through microporous polyethylene/ $\mathrm{CaCO}_{3}$ films. Colloids and Surfaces, Part A: Physicochemical and Engineering Aspects, 187-188, 483-491 (2001).

[5] Bucknall C. B.: Toughened Plastics. Applied Science, London (1977).

[6] Pukánszky B., van Es M., Maurer F. H. J., Vörös G.: Micromechanical deformations in particulate filled thermoplastics- volume strain measurements. Journal of Material Science, 29, 2350-2358 (1994).

[7] Farris R. J.: The influence of vacuole formation on the response and failure of filled elastomers. Transactions of the Society of Rheology, 12, 315-334 (1968).

[8] van Hartingsveldt E. A. A., van Aartsen J. J.: Interfacial debonding in polyamide-6/glass bead composites. Polymer, 30, 1984-1991 (1989).

[9] Boven G., Folkersma R., Challa G., Scouten A. J., Bosma M.: Polymer-filler interactions in poly(vinyl chloride) filled with glass beads: effect of grafted poly(methyl methacrylate). Polymer, 33, 83-88 (1992).

[10] Dubnikova I. L., Muravin D. K., Oshmyan V. G.: Debonding and fracture of particulate-filled isotactic polypropylene. Polymer Engineering and Science, 37, 1301-1313 (1997).

[11] Meddad A., Fisa B.: Stress-strain behavior and tensile dilatometry of glass bead-filled polypropylene and polyamide 6. Journal of Applied Polymer Science, 64, 653-665 (1997).

[12] Asp L. E., Sjögren B. A., Berglund L. A.: Prediction of failure initiation in polypropylene with glass beads. Polymer Composites, 18, 9-15 (1997).

[13] Sjögren B. A., Berglund L. A.: Failure mechanisms in polypropylene with glass beads. Polymer Composites, 18, 1-8 (1997).

[14] Tsui C. P., Tang C. Y., Lee T. C.: Tensile properties and damage behaviors of glass-bead-filled modified polyphenylene oxide under large strain. Polymer Composites, 22, 742-751 (2001). 
[15] Lazzeri A., Thio Y. S., Cohen R. E.: Volume strain measurements on $\mathrm{CaCO}_{3}$ /polypropylene particulate composites: The effect of particle size. Journal of Applied Polymer Science, 91, 925-935 (2004).

[16] Thio Y. S., Argon A. S., Cohen R. E.: Role of interfacial adhesion strength on toughening polypropylene with rigid particles. Polymer, 45, 3139-3147 (2004).

[17] Vollenberg P., Heikens D., Ladan H. C. B.: Experimental determination of thermal and adhesion stress in particle filled thermoplasts. Polymer Composites, 9, 382-388 (1988).

[18] Vollenberg P. H. T.: The mechanical behaviour of particle filled thermoplastics. PhD Thesis, Eindhoven University of Technology (1987).

[19] Meddad A., Fisa B.: A model for filler-matrix debonding in glass-bead-filled viscoelastic polymers. Journal of Applied Polymer Science, 65, 2013-2024 (1997).

[20] Pukánszky B., Vörös G.: Mechanism of interfacial interactions in particulate filled composites. Composite Interfaces, 1, 411-427 (1993).

[21] Pukánszky B., Fekete E.: Adhesion and surface modification. Advances in Polymer Science, 139, 109-153 (1999).

[22] Pukánszky B., Fekete E.: Aggregation tendency of particulate fillers: Determination and consequences. Polymer and Polymer Composites, 6, 313-322 (1998).

[23] Fekete E., Molnár Sz., Kim G-M., Michler G. H., Pukánszky B.: Aggregation, fracture initiation, and strength of $\mathrm{PP} / \mathrm{CaCO}_{3}$ composites. Journal of Macromolecular Science Physics, Part B: Physics, 38, 885899 (1999).

[24] Ess J. W., Hornsby P. R.: Characterization of distributive mixing in thermoplastic compositions. Polymer Testing, 6, 205-218 (1986).
[25] Kim G-M., Lee D-H.: FEG-ESEM investigation of micromechanical deformation processes in ultrafine monospherical $\mathrm{SiO}_{2}$ particle-filled polymer composites. Journal of Applied Polymer Science, 82, 785-789 (2001).

[26] Kim G-M., Michler G. H.: Micromechanical deformation processes in toughened and particle filled semicrystalline polymers: Part 2. Model representation for micromechanical deformation processes. Polymer, 39, 5699-5703 (1998).

[27] Nilsen L. E., Landel R. F.: Mechanical properties of polymers and composites. Marcel Dekker, New York (1974).

[28] Kolarík J., Lednický F., Pukánszky B.: Ternary composites polypropylene/ elastomer/filler: Structure and elastic properties. in 'Proceedings of $6^{\text {th }} \mathrm{ICCM} / 2^{\text {nd }}$ ECCM, London, United Kingdom' Vol 1., 452-461 (1987).

[29] Parsons E. M., Boyce M. C., Parks D. M., Weinberg M.: Three-dimensional large-strain tensile deformation of neat and calcium carbonate-filled high-density polyethylene. Polymer, 46, 2257-2265 (2005).

[30] G'Sell C., Hiver J. M., Dahoun A., Souahi A.: Videocontrolled tensile testing of polymers and metals beyond the necking point. Journal of Materials Science, 27, 5031-5039 (1992).

[31] Dubnikova I. L., Berezina S. M., Antonov A. V.: The effect of morphology of ternary-phase polypropylene/glass bead/ethylene-propylene rubber composites on the toughness and brittle-ductile transition. Journal of Applied Polymer Science, 85, 1911-1928 (2002). 